\title{
Desenvolvimento de um inquérito para avaliação da ingestão alimentar de grupos populacionais
}

\author{
Development of a questionnaire to assess \\ food intake of population groups
}

Regiane Lopes de SALES ${ }^{1}$

Margarida Maria Santana SILVA²

Neuza Maria Brunoro COSTA ${ }^{2}$

Marilene Pinheiro EUCLYDES ${ }^{2}$

Vivian Fernandes ECKHARDT ${ }^{3}$

Cláudia Márcia Antunes RODRIGUES ${ }^{4}$

Adelson Luiz Araújo TINÔCO²

\section{RES U M O}

\section{Objetivos}

Este trabalho procurou desenvolver um instrumento para obtenção de dados relativos ao consumo alimentar, de caráter qualitativo e quantitativo, para avaliar a ingestão dietética em estudos populacionais.

\section{Métodos}

Inicialmente foram coletados dados sobre a ingestão alimentar de uma amostra representativa de moradores da cidade de Viçosa $(n=119)$. Para conhecer os alimentos mais consumidos e seus porcionamentos, foram utilizados os métodos recordatório de $24 \mathrm{~h}$ e pesagem direta. A entrevista ocorreu no horário do almoço ou jantar, com pesagem de todos os alimentos constantes na refeição e de porções referidas como consumidas nas últimas 24h, usou-se também álbum fotográfico como auxiliar na estimativa do peso da porção.

\section{Resultados}

A partir dos 60 alimentos mais consumidos e dos porcionamentos identificados, foi desenvolvido um inquérito baseado no método Freqüência de Consumo Alimentar semi-quantitativo, com 65 itens e um álbum fotográfico de alimentos. Realizou-se um estudo piloto para avaliação desta metodologia em uma sub-amostra $(n=34)$. Para comparação entre os dois métodos, avaliaram-se o valor energético e os macronutrientes, utilizando o coeficiente de Pearson e o teste " $t$ " de Student, tendo-se obtido: $r=0,53$ para energia, $r=0,43$ para proteína,

\footnotetext{
$\overline{1}$ Doutoranda em Ciência e Tecnologia de Alimentos, Universidade Federal de Viçosa. Viçosa, MG, Brasil.

2 Departamento de Nutrição e Saúde, Universidade Federal de Viçosa. Av. P.H. Rolfs, /sn, Campus Universitário, 36571-000, Viçosa, MG, Brasil. Correspondência para/Correspondence to: M.M.S. SILVA. E-mail: <msilva@ufv.br>.

${ }^{3}$ Instituto Nacional de Colonização e Reforma Agrária. Campo Grande, MS, Brasil.

4 Centro Universitário do Espírito Santo. Colatina, ES, Brasil.
} 
$r=0,58$ para lipídeos e $r=0,21$ para carboidratos. Não foi detectada diferença significante entre os dois métodos para proteína e lipídeos, pelo teste " $t$ " $(\alpha=0,05)$.

\section{Conclusão}

O inquérito desenvolvido mostrou-se um instrumento promissor para inquéritos populacionais, no entanto, são necessários maiores estudos para averiguar as limitações e os possíveis desdobramentos do inquérito para atingir toda a população.

Termos de indexação: avaliação nutricional; consumo alimentar; ingestão alimentar; inquéritos nutricionais.

\section{A B S T R A C T}

\section{Objective}

This study sought to develop an instrument to obtain and assess data on the qualitative and quantitative food intake of population groups.

\section{Methods}

Initially, data on food intake of a representative sample of inhabitants of Viçosa $(n=119)$ were collected. The 24-hour recall and direct weight were used to determine the most consumed foods and their portions. Interview was done at lunch or dinner where all foods and portions mentioned in the previous 24 hours were weighed; a food picture album was used to aid in determining the weight of the portion.

\section{Results}

From the 60 most frequently consumed foods and the identified portions, a questionnaire based on the semi-quantitative Food Intake Frequency method, with 65 items and a food picture album were developed. A pilot study was done to assess this methodology in a subsample $(n=34)$. To compare the two methods, the energy value and macronutrients were assessed, using the Pearson coefficient and the Student's " $t$ " test and the values obtained were $r=0.53$ for energy, $r=0.43$ for proteins, $r=0.58$ for lipids and $r=0.21$ for carbohydrates. A significant difference was not detected by the Student's " $t$ " test for proteins and lipids between the two methods ( $\alpha=0.05)$.

\section{Conclusion}

The questionnaire was proven to be a promising method for population surveys, yet more studies are necessary to determine its limitations and possibly expand the questionnaire in order to cover the entire population.

Indexing terms: nutrition assessment; food consumption; eating; nutrition survey.

\section{N T R O D U Ç Ã O}

Apesar do inegável impacto das ações básicas de saúde, o Brasil ainda apresenta um quadro de persistência da morbimortalidade infantil, caracterizado por doenças infecto-contagiosas e desnutrição, típico de populações carentes. Além disso, observa-se o aumento da prevalência de doenças crônicas não-transmissíveis, destacando-se a obesidade, um fenômeno característico de populações em processo de enriquecimento ${ }^{1}$. Tal situação reflete um perfil epidemiológico complexo, que exige acompanhamento competente e minucioso, a fim de subsidiar políticas de intervenção.
Para uma efetiva avaliação nutricional, a verificação do padrão de consumo de alimentos de uma população é essencial, além de se tratar de informação básica para o direcionamento de políticas públicas de diversos setores, como o armazenamento e o transporte de alimentos, o comércio e a saúde (para definir as recomendações nutricionais, pesquisa epidemiológica sobre a relação entre alimentação e doenças, identificar áreas endêmicas, entre outras)².

Essas informações são escassas ainda hoje, a melhor fonte de dados no País data de 1974/1975, proveniente do Estudo Nacional sobre Despesa Familiar (ENDEF), realizado pelo Instituto Brasileiro de Geografia e Estatística (IBGE) ${ }^{3}$, em 
que foram entrevistados 55 mil domicílios. Mas o alto custo operacional inviabilizou a repetição da sua metodologia, altamente trabalhosa. Para sua realização, os entrevistadores pesquisavam cada domicílio, ao longo de sete dias consecutivos, e eram feitas tantas visitas quantas fossem as refeições principais da família. Os alimentos comprados no dia e os que seriam consumidos na refeição seguinte eram pesados e sacos plásticos eram deixados para que fossem guardados os resíduos, as sobras e os desperdícios, para posterior pesagem. Depois, uma série de cálculos eram efetuados para transformar em índices que pudessem ser comparados entre as famílias e as necessidades nutricionais ${ }^{4}$.

Após o ENDEF, foram realizadas três Pesquisas de Orçamentos Familiares (POF) em 1987-1988, 1995-1996 e 2002-2003 de menor abrangência (13 611, 16014 e 48470 domicílios, respectivamente). Foram coletados dados sobre despesas familiares com gêneros alimentícios, porém elas têm como objetivo principal estimar os índices de preços e não o consumo alimentar individual. As POF contemplaram o consumo sob a ótica do orçamento domiciliar, ou seja, se detiveram nos gastos monetários da família referentes à alimentação $0^{5-7}$. Elas não permitem avaliar o consumo individual, ocupando uma posição intermediária entre as folhas de balanço de alimentos, que descrevem a disponibilidade nacional de alimentos, e os inquéritos desenhados para avaliação do consumo alimentar. Apesar dessa limitação, fornecem dados sobre a alimentação e se constituem em importante banco de informações que foram utilizadas em vários estudos sobre hábitos alimentares da população brasileira $^{8-10}$.

Em 1989, o extinto Instituto Nacional de Alimentação e Nutrição (INAN) promoveu a Pesquisa Nacional de Saúde e Nutrição (PNSN), uma parceria entre o Ministério da Saúde, o IBGE e o Instituto de Pesquisa Econômica Aplicada (IPEA), que utilizou somente o indicador antropométrico, excluindo o indicador dietético, um instrumento essencial para a compreensão da etiologia dos desvios nutricionais da população'11. Em 1996 foi realizada a segunda PNSN, que descreveu informações sobre o estado nutricional de mães e de crianças, menores de cinco anos no País, excluindo as outras faixas etárias.

Entre os anos de 1996 e 1997, foi realizado o Estudo Multicêntrico de Consumo Alimentar (EMCA) de 1996, em cinco grandes centros (Goiânia, Distrito Federal, Campinas, Ouro Preto, Rio de Janeiro e Curitiba), empregando-se duas metodologias para análise do consumo, o Inquérito de Consumo Alimentar Familiar (INCAF) ${ }^{12}$ e o questionário de freqüência alimentar semi-quantitativo, adaptado para a dieta brasileira por Sichieri \& Everhart ${ }^{13}$, associado a um álbum fotográfico. Foi detectado, assim, o perfil alimentar dos indivíduos com 19 anos ou mais. O EMCA1996, posteriormente, foi estendido a Belém e a Brasília.

Esses estudos foram os únicos de abrangência nacional. Eles utilizaram metodologias diferentes, reflexo da dificuldade de estabelecimento de um padrão-ouro para avaliação nutricional de populações ${ }^{14}$.

Devido ao alto custo conferido pela utilização de técnicas mais apuradas, elevado tempo de aplicação, necessidade de profissionais especializados em todas as etapas da pesquisa, maior complexidade na fase de análise e divulgação dos resultados, a periodicidade e a agilidade dessas pesquisas são reduzidas ${ }^{14}$.

Diante desse quadro, várias iniciativas vêm tentando preencher a lacuna deixada pela carência de um sistema de vigilância nutricional ativo no País, desenvolvendo questionários para aplicação em grupos específicos, como o elaborado por Bonomo et al. ${ }^{15}$, Furlan-Viebig \& Pastor-Valero ${ }^{16}$ e Colucci ${ }^{17}$.

Assim, o objetivo deste trabalho foi desenvolver um instrumento para avaliação da ingestão alimentar de grupos populacionais, menos susceptível aos erros de interpretação, de relativo baixo custo e que contribua para a realização de levantamentos do consumo alimentar. 


\section{MÉTODOS}

Os alimentos e preparações consumidos com maior freqüência na microrregião de Viçosa foram, inicialmente, identificados a partir de fontes secundárias (dados não publicados) e, devido à insuficiência dos dados, realizou-se a pesquisa de campo para o estabelecimento do tamanho das porções ingeridas.

A determinação do tamanho da amostra seguiu a metodologia proposta por Lwanga \& Lemessow $^{18}$, a partir de dados fornecidos pelo senso do IBGE referentes à pirâmide etária da população de Viçosa. A amostra estabelecida foi de 119 pessoas (Tabela 1), estratificada por estágios de vida e gênero, proporcionalmente à pirâmide etária da cidade de Viçosa, selecionada de acordo com o método de Redes Sociais, também conhecido como "Bola de Neve"19.

De acordo com a metodologia do IBGE, a amostragem deveria incluir crianças a partir do nascimento, porém, considerando que nesse estágio de vida grande porcentagem delas se encontram em processo de amamentação, optou-se pela exclusão de crianças menores de um ano,

Tabela 1. Distribuição da população por faixa etária e sexo, de acordo com método recordatório/pesagem. Viçosa, 1996/1997.

\begin{tabular}{lccc}
\hline Faixas etárias & Masculino & Feminino & Total \\
\hline 1 a 4 anos & 4 & 4 & 8 \\
5 a 9 anos & 4 & 4 & 8 \\
10 a 14 anos & 4 & 4 & 8 \\
15 a 19 anos & 5 & 5 & 10 \\
20 a 24 anos & $7 *$ & 4 & 11 \\
25 a 29 anos & 4 & 4 & 8 \\
30 a 34 anos & 3 & 3 & 6 \\
35 a 39 anos & 3 & 3 & 6 \\
40 a 44 anos & 3 & 3 & 6 \\
45 a 49 anos & 3 & 3 & 6 \\
50 a 54 anos & 3 & 3 & 6 \\
55 a 59 anos & 3 & 3 & 6 \\
60 a 64 anos & 3 & 3 & 6 \\
65 a 69 anos & 3 & 3 & 6 \\
70 a 74 anos & 3 & 3 & 6 \\
75 a79 anos & 3 & 3 & 119 \\
80 e mais & 3 & 3 & 58 \\
\hline Total & 61 & & 6
\end{tabular}

*Excedentes (3). uma vez que precisar quantitativamente a ingestão do leite materno demandaria metodologia específica e invasiva que, provavelmente, afetaria o consumo real desse alimento, contrariando os objetivos desta pesquisa.

O consumo alimentar foi avaliado por intermédio da aplicação do método recordatório de $24 \mathrm{~h}$, aliado à pesagem direta, visando à obtenção de dados qualitativos e quantitativos para determinação dos tamanhos das porções, que serviram, posteriormente, de base para a formulação do inquérito.

Foram realizadas entrevistas únicas, no período de fevereiro a julho de 1997, em dias úteis, no momento do almoço ou jantar dos entrevistados, nas quais todos os alimentos ingeridos e as sobras alimentares foram pesados em tempo real de consumo. O recordatório de 24h foi aplicado após o almoço/jantar, identificando todos os alimentos consumidos nas últimas 24h, fazendo a pesagem direta dos alimentos encontrados. Para a pesagem, utilizou-se uma balança digital eletrônica com capacidade $2000 \mathrm{~g}$ e sensibilidade de $0,1 \mathrm{~g}$.

Alguns alimentos não puderam ser pesados, por motivos diversos, como o de ingestão fora do ambiente domiciliar; por não constar na casa, no momento da entrevista; consumo total da preparação, não restando amostras para identificação e pesagem. Para esses alimentos, o peso foi estimado com o auxílio de álbuns de fotografias desenvolvidos por Trigo ${ }^{20}$ e Zabotto ${ }^{21}$.

Também foram obtidas informações sobre os ingredientes que compõem as preparações de forma qualitativa e quantitativa, fornecidas pelo responsável pela elaboração das preparações, assim como as receitas das mesmas, para melhor análise dos dados.

Os dados foram compilados e analisados utilizando-se o programa computacional Diet Pro (Agromídia Ltda, Viçosa, MG).

Com base nos alimentos e porcionamentos identificados, foi desenvolvido um inquérito de consumo alimentar (Anexo), contendo os alimentos mais consumidos pela amostra entrevistada, 
e um álbum fotográfico contendo os mesmos alimentos, para auxilio na identificação das porções consumidas.

Para definir os tamanhos das porções, utilizou-se a média ponderada dos picos de porções de maior ocorrência, identificados no inquérito dietético realizado. A fórmula utilizada para tal foi: $\underline{\Sigma(P \times O)}=$ tamanho da porção.

\section{$\Sigma O$}

Em que:

$\mathrm{O}=$ ocorrência ( $\mathrm{se} \geq 2$ ) e $\mathrm{P}=$ porção.

Visando oferecer maior gama de opções de porcionamentos, foram utilizadas cinco porções a partir desse critério elaborado.

Para o registro fotográfico dos alimentos foram utilizados equipamento digital e utensílios convencionais, como copo americano, copo duplo, colher de sopa, colher de sobremesa, pratos lisos e pratos de sobremesa. Para reproduzir as porções definidas no inquérito, os alimentos foram pesados em balança digital com sensibilidade de $1 \mathrm{~g}$. E para que fosse preservada a noção do tamanho real das preparações, utilizou-se uma caixa de fósforos, no intuito de ajudar o entrevistado a identificar a porção consumida e comparar à reproduzida no inquérito de forma mais objetiva e precisa ${ }^{21}$.

As fotografias foram dispostas segundo grupo de alimentos, as porções diversificadas em cinco tamanhos, em ordem crescente: A, B, C, D e E.

Para indicar a freqüência de consumo, foi elaborado um código, inserido no inquérito (7-6-5-4-3-2-1-T-Q-R), sendo os números correspondentes ao número de dias da semana em que o alimento é consumido; T e Q indicam a freqüência mensal - 3 e 2 vezes/ mês, respectivamente; $\mathrm{R}$ os alimentos que raramente são consumidos.

\section{Estudo piloto}

O inquérito foi submetido a estudo piloto com uma sub-amostra de 34 pessoas, constituída por voluntários que participaram da entrevista anterior com a pesagem direta e o recordatório 24h, no período de março a julho de 1998.

Identificou-se a ingestão de proteínas, carboidratos, lipídeos e energia, a qual foi comparada com os dados do método anteriormente aplicado. As análises da composição química foram efetuadas utilizando-se o programa computacional Diet Pro.

Os dados de consumo dos macronutrientes do inquérito foram comparados aos obtidos pelo método recordatório $24 \mathrm{~h}$, por meio do coeficiente de correlação de Pearson e do teste " $t$ " de Student $(\alpha=0,05)$, além de análise gráfica utilizando-se o programa estatístico Statistica, versão 5 .

\section{RESULTADOSE DISCUSSÃO}

O inquérito (Anexo) reuniu os métodos freqüência qualitativa e quantitativa de consumo alimentar e recordatório $24 \mathrm{~h}$, além do recurso auxiliar do álbum fotográfico ("avaliando o consumo alimentar por fotos" $)^{22}$.

As médias dos valores de energia, proteína, lipídeo e carboidrato, e as respectivas correlações do recordatório $24 \mathrm{~h}$ e do inquérito desenvolvido estão apresentadas na Tabela 2.

Tabela 2. Análise estatística de energia e macronutrientes do recordatório/pesagem e inquérito proposto, por faixa etária. Viçosa, MG 1996/1998.

\begin{tabular}{|c|c|c|c|c|c|c|c|c|}
\hline \multirow[b]{2}{*}{ Faixa etária } & \multicolumn{2}{|c|}{ Energia } & \multicolumn{2}{|c|}{ Proteína } & \multicolumn{2}{|c|}{ Lipídeo } & \multicolumn{2}{|c|}{ Carboidrato } \\
\hline & $\begin{array}{c}\text { Recordatório/ } \\
\text { pesagem }\end{array}$ & $\begin{array}{l}\text { Inquérito } \\
\text { proposto }\end{array}$ & $\begin{array}{c}\text { Recordatório/ } \\
\text { pesagem }\end{array}$ & $\begin{array}{l}\text { Inquérito } \\
\text { proposto }\end{array}$ & $\begin{array}{c}\text { Recordatório/ } \\
\text { pesagem }\end{array}$ & $\begin{array}{l}\text { Inquérito } \\
\text { proposto }\end{array}$ & $\begin{array}{c}\text { Recordatório/ } \\
\text { pesagem }\end{array}$ & $\begin{array}{l}\text { Inquérito } \\
\text { proposto }\end{array}$ \\
\hline Média & 2339,30 & 2002,30 & 94,55 & 77,56 & 89,66 & 67,79 & 297,26 & 274,00 \\
\hline DP & 585,24 & 554,62 & 36,35 & 20,37 & 31,41 & 27,38 & 63,43 & 73,55 \\
\hline r & \multicolumn{2}{|c|}{$0,525^{0,04}$} & \multicolumn{2}{|c|}{$0,43^{0,13}$} & \multicolumn{2}{|c|}{$0,58^{0,02}$} & \multicolumn{2}{|c|}{$0,21^{0,45}$} \\
\hline $\mathrm{t}(\propto=0,05)$ & \multicolumn{2}{|l|}{0,03} & \multicolumn{2}{|c|}{0,06} & \multicolumn{2}{|l|}{0,02} & \multicolumn{2}{|c|}{$0,31^{*}$} \\
\hline
\end{tabular}

Nota: DP=desvio-padrão; $r=$ coeficiente de correlação; *representava 'valor estatisticamente não significante'. 
De acordo com Willet ${ }^{23}$, por não existir um método que seja padrão-ouro em inquérito dietético para avaliar um novo método, uma das formas é realizar uma validação relativa, ou seja, compará-lo com outro método que seja considerado válido e de boa reprodutibilidade. E uma análise adotada na validação relativa de métodos dietéticos é o coeficiente de correlação de Pearson (r). Em inquéritos dietéticos, segundo Willet ${ }^{23} \mathrm{e}$ Riboli \& Kaaks ${ }^{24}$, é aceitável que este coeficiente esteja compreendido num valor entre 0,4 e 0,7.

Com exceção dos carboidratos, que apresentaram uma correlação não significante $(r=0,21)$, os demais nutrientes revelam valores das estimativas dentro do intervalo considerado para as correlações (energia, $r=0,53$; proteína, $r=0,43$ e lipídeos, $r=0,58)$. A média total das correlações foi de 0,44 , aceitável para validação de inquéritos dietéticos. Esse valor é semelhante ao de outro trabalho, realizado por Sichieri \& Everhart ${ }^{13}$, que desenvolveram um questionário de freqüência alimentar validado com o recordatório $24 \mathrm{~h}$ em que, para os mesmos nutrientes, apresentaram média total das correlações de $r=0,41$. Crispim ${ }^{25}$ realizou uma validação relativa do inquérito desenvolvido, quatro anos mais tarde, com uma metodologia semelhante (aplicando o recordatório $24 \mathrm{~h}$ não somente uma, mas em quatro vezes, em diferentes datas) encontrou uma boa reprodutibilidade, com média total das correlações de $r=0,52$, em que foram avaliados os macro e micronutrientes.

Pelo teste " $t$ ", com valores de $p$ significantes ao nível de $5 \%$ de probabilidade, o inquérito desenvolvido apresentou os valores de 0,03 para energia; 0,06 para proteína; 0,02 para lipídeo e 0,31 para carboidratos. As proteínas e os carboidratos não apresentaram significância, no entanto, deve-se considerar que apenas um dia de recordatório $24 \mathrm{~h}$ foi aplicado nesta pesquisa, o ideal seria repeti-lo por mais duas ou três vezes, em diferentes datas, para minimizar os erros quanto à variação intra e inter individual, e incluir o fator sazonalidade, que pode influenciar nos tipos de alimentos que são relatados nos inquéritos ${ }^{19}$.
As Figuras 1 e 2 mostram a média e o desvio-padrão dos métodos para energia, carboidrato, proteína e lipídeo. Observa-se que o método desenvolvido apresenta o mesmo comportamento em relação ao recordatório $24 \mathrm{~h}$, apesar de subestimar a quantidade ingerida de energia e dos nutrientes supracitados, essa diferença se mostrou insignificante para energia e lipídeos.

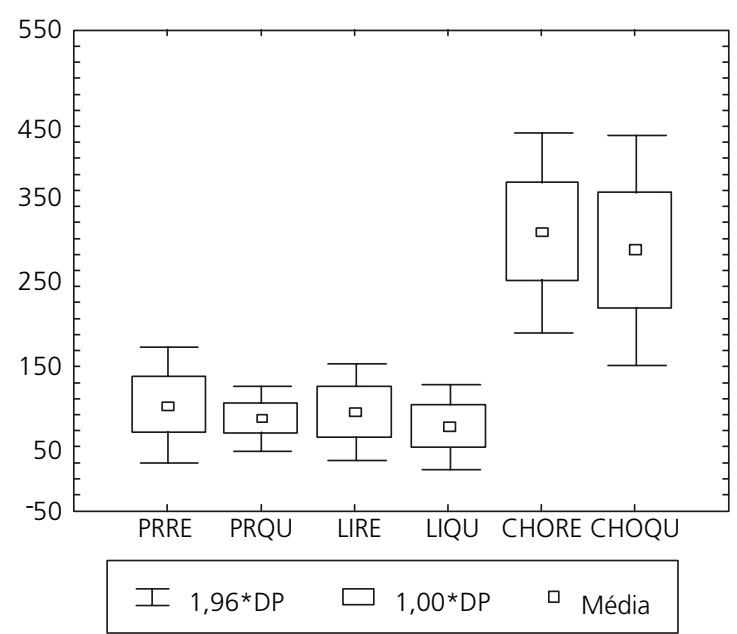

Figura 1. Consumo de proteína, lipídeo e carboidrato, recordatório/pesagem e Inquérito proposto. Viçosa, 19961998.

Nota: PRRE=proteína, recordatório/pesagem; PRQU=proteína, Inquérito proposto; LIRE=lipídeo, recordatório; LIQU=lipídeo, inquérito; $\mathrm{CHORE}=$ carboidrato. Recordatório; $\mathrm{CHOQU}=$ carboidrato, inquérito.

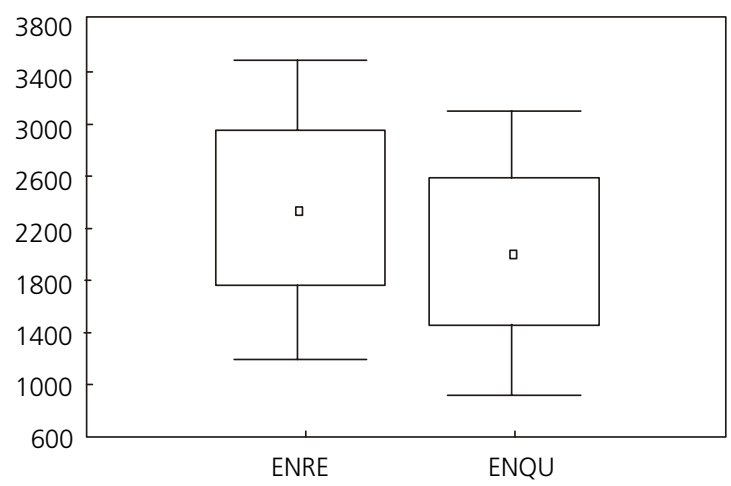

Figura 2. Consumo de energia, registro/pesagem e inquérito proposto. Viçosa, 1996-1998.

Nota: ENRE=energia, recordatório/pesagem; ENQU=energia, Inquérito proposto. 
De acordo com Hansson et al. ${ }^{26}$, uma definição prévia do tamanho da porção pode prejudicar a estimativa real do consumo alimentar, por não permitir maior variabilidade de respostas dos entrevistados. No entanto, dada a necessidade de obter um estudo relativamente de baixo custo e de alta precisão, a utilização de fotos em inquéritos dietéticos agiliza a aplicação do método e reduz os erros de interpretação do entrevistador e do entrevistado ${ }^{27}$. Outras vantagens são: os hábitos alimentares não são alterados pela presença do entrevistador no momento da refeição, o tempo de coleta de dados é menor, e vários indivíduos podem ser investigados em um mesmo dia, sem prejuízo para a qualidade dos dados ${ }^{19}$. $O$ álbum fotográfico desenvolvido apresenta duas fotos de cada porção do alimento em diferentes ângulos, para permitir melhor detalhamento do tamanho da porção, uma fotografia retratando a parte superior do alimento e outra na diagonal lateral, para aumentar a precisão entre a mesma e o alimento consumido, diferentemente dos demais trabalhos publicados que apresentam uma única fotografia da porção 20,21.

O inquérito desenvolvido possui 34 itens, dos 73 apresentados no questionário elaborado por Sichieri \& Everath ${ }^{13}$, aplicado no Rio de Janeiro, e contém ainda 30 dos 48 itens do desenvolvido por Colucci ${ }^{17}$, para crianças de 2 a 5 anos. A maioria dos questionários desenvolvidos são destinados a grupos específicos, como crianças ${ }^{17}$, adolescentes $^{28}$, ou para a identificação de riscos para doenças crônicas não transmissíveis, entre outros ${ }^{16}$. Isto permite maior validade dos mesmos, pela maior especificidade apresentada nos alimentos que os compõem ${ }^{27}$. Porém, este não foi o objetivo inicial deste trabalho.

Entretanto, elaborar um questionário extenso diminui a sua eficiência27, e, por outro lado, abranger todos os estágios de vida, condições socioeconômicas e de saúde, num único questionário resumido, parece contraditório.

A habilidade do método para estimar com acurácia os desvios nutricionais relacionados ao consumo alimentar depende do delineamento do estudo, da seleção da amostra, da heterogeneidade do padrão alimentar da mesma, das variáveis de confundimento, além de uma série de erros a que está sujeito, como a habilidade do entrevistador, a variação intra e inter individual, os fatores ambientais e comportamentais que podem predispor a um determinado desvio nutricional ${ }^{29}$.

Assim, fazem-se necessários maiores estudos para averiguar as limitações e os possíveis desdobramentos do inquérito para atingir toda a população. Como aconteceu com o ENDEF, pesquisas de alto custo são de difícil reprodução, impossibilitando o monitoramento das condições de saúde da população. A aplicação dos métodos de referência (recordatório $24 \mathrm{~h}$ e pesagem direta) exige um alto investimento (para treinamento de entrevistadores, compra de balanças, deslocamento, analistas, entre outros), a metodologia desenvolvida aqui apresentou uma boa reprodutibilidade neste estudo.

\section{CONCLUSÃO}

O inquérito desenvolvido foi um bom instrumento para mensurar o valor de energia e lipídeo neste estudo, podendo ser utilizado em análises de ingestão alimentar de populações. Contudo, novos estudos precisam ser realizados com a metodologia utilizada, no intuito de verificar a reprodutibilidade e proceder validação do instrumento desenvolvido, uma vez que o presente trabalho é insuficiente para inferências de caráter mais abrangente.

\section{REFERÊ NCIAS}

1. Pinheiro ARO, Freitas SFT, Corso ACT. Uma abordagem epidemiológica da obesidade. Rev Nutr. 2004; 17(4):523-33.

2. Galeazzi MAM, Domene SMA, Sichieri R. Estudo multicêntrico sobre consumo alimentar. Cad Debate. 1997; (Volume especial):11-60.

3. Instituto Brasileiro de Geografia e Estatística. Estudo Nacional de despesa familiar. Consumo 
alimentar e antropometria. Dados preliminares. v.1, T.1, Pte. 2 (região V) e v. 1, T.1, Pte. 3 (regiões II e IV). Rio de Janeiro; 1977.

4. Vasconcellos MT, Anjos LA. Taxa de adequação (ingestão/ requerimento) de energia como indicador do estado nutricional das famílias: uma análise crítica dos métodos aplicados em pesquisas de consumo de alimentos. Cad Saúde Pública. 2001; 17(3):581-93.

5. Instituto Brasileiro de Geografia e Estatística. Pesquisa do orçamento familiar. POF 1987/88. Rio de Janeiro; 1991. v.1.

6. Instituto Brasileiro de Geografia e Estatística. Pesquisa do orçamento familiar. POF 1995-1996: primeiros resultados. Rio de Janeiro; 1997.

7. Instituto Brasileiro de Geografia e Estatística. Pesquisa de orçamentos familiares. POF 20022003: análise da disponibilidade domiciliar de alimentos e do estado nutricional no Brasil. Rio de Janeiro; 2004.

8. Monteiro CA, Mondini L, Costa RBL. Mudanças na composição e adequação nutricional da dieta familiar nas áreas metropolitanas do Brasil (1988-1996). Rev Saúde Pública. 2000; 34(3) 251-58.

9. Szarfarc SC, Monteiro CA, Meyer M, Tudisco ES, Reis IM. Estudo das condições de saúde das crianças do município de São Paulo, SP (Brasil), 1984/1985. X Consumo alimentar. Rev Saúde Pública. 1988; 22(4)226-72.

10. Levy-Costa RB, Sichieri R, Pontes NS, Monteiro CA Disponibilidade domiciliar de alimentos no Brasil: distribuição e evolução (1974-2003). Rev Saúde Pública. 2005; 39(4):530-40.

11. Brasil. Ministério da Saúde. Instituto Nacional de Alimentação e Nutrição. Pesquisa nacional sobre saúde e nutrição: resultados preliminares. Brasília; 1990.

12. Galeazzi MAM, Bonvino H, Lourenço F, Vianna RPT. Inquérito de consumo familiar de alimentos: Metodologia para identificação de famílias de risco alimentar. Cad Debate. 1996; 4:57

13. Sichieri R, Everhart JE. Validity of a brazilian food frequency questionary against dietary recalls and estimated energy intake. Nutr Res. 1998; 18(10):1649-59.

14. Cintra IP, von Der Heyde MED, Schimitz BAS, Franceschini SCC, Taddei JA, Sigulem DM. Métodos de inquéritos dietéticos. Cad Nutr. 1997; 13:11-23.

15. Bonomo E, Laiaffa WT, César CC, Lopes ACS, Costa MFL. Consumo alimentar da população adulta segundo perfil sócio-econômico e demográfico: Projeto Bambui. Cad Saúde Pública. 2003; 19(5):1461-71.
16. Furlan-Viebig R, Pastor-Valero M. Desenvolvimento de um questionário de freqüência alimentar para o estudo de dieta e doenças não-transmissíveis. Rev Saúde Pública. 2004; 38(4):581-4.

17. Colucci ACA. Desenvolvimento de um questionário de freqüência alimentar para avaliação do consumo alimentar de crianças de 2 a 5 anos de idade. Rev Bras Epidemiol. 2004; 7(4):393-401.

18. Lwanga SK, Lemessow S. Sample size determinations in health studies; a pratical manual. Geneva: World Health Organization; 1982.

19. Weiss RS. Learning from stranger: the art and method of qualitative interview studies. New York: The Free Press; 1994.

20. Trigo M. Estudo da metodologia de inquérito dietético: validade do método recordatório de 24 horas [tese]. São Paulo: Faculdade de Saúde Pública, Universidade de São Paulo; 1993.

21.Zabotto CB, Vianna RPT, Gil MF. Registro fotográfico para inquéritos dietéticos: utensílios e porções. Goiânia: Nepa-Unicamp; 1996.

22. Sales RL, Silva MMS, Costa NMB. Avaliando o consumo alimentar por fotos [CD-Rom]. Viçosa: UFV; 2004.

23. Willett WC. Nutritional epidemiology, monographs in epidemiology and bioestatistic, Oxford: Oxford University Press; 1990. v.15.

24. Riboli E, Kaaks R. The EPIC projet: rationale and study design. Int J Epidemiol. 1997; 23(Suppl.I): S6-S14.

25. Crispim S. Validação relativa de um questionário de freqüência alimentar para avaliação da ingestão dietética em adultos residentes no município de Viçosa - Minas Gerais [dissertação]. Viçosa: Universidade Federal de Viçosa; 2004.

26. Hansson LM, Galanti MR, Bergströn R. Factors affecting reproducibility of dietary reports using food frequency questionnaires. Eur J Clin Nutr. 2000; 54(8):658-64.

27. Nelson M, Atkinson M, Darbyshire S. Food photography II: use of food photographys for estimating portion size and nutrient content of meals. Br J Nutr. 1996; 76(1):31-49.

28. Binghan SA, Cassidy A, Cole TJ, Welch A, Runswick $S A$, Black $A E$, et al. Validation of weighed records and other methods of dietary assessment using the $24 \mathrm{~h}$ urine nitrogen and other biological markers. Br J Nutr. 1995; 73(4):531-50

29. Nelson PM. The validation of dietary assessment. In: design concepts in nutrition epidemiology. 2nd ed. Oxford: Oxford University Press; 1997.

Recebido em: 4/6/2003

Versão final reapresentada em: 9/9/2005 Aprovado em: 19/10/2005 


\section{ANEXO}

INQUÉRITO DE CONSUMO ALIMENTAR

1 DADOS PESSOAIS

Data:

Primeiro nome:

Endereço:

Correspondendo à: D S T Q Q S S

Cidade:

$\mathrm{N}^{\mathrm{O}}$ Profissão:

Nascimento: 1

Bairro:

2 DADOS ANTROPOMÉTRICOS E AVALIAÇÃO NUTRICIONAL

Atividade Física

kg. Altura: $\mathrm{cm}$ IMC $\%$

O Repouso

O Muito Leve

O Leve

O Moderada

O Intensa

História Clínica
$\square$ Enfermidade renal
$\square$ Enfermidade Cardiovascular
$\square$ Enfermidade Hepática
$\square$ Enteropatias

$\square$ Diabetes

$\square$ Nenhum

$\square$ Outro(s) Qual(is):

\section{INSTRUÇÕES}

Este questionário é sobre o consumo qualitativo e quantitativo de alimentos

Indique o número de dias da semana que os alimentos constituintes da dieta normal são consumidos, conforme o exemplo indicado abaixo:

Se determinado alimento é consumido todos os dias, marque 7

Se é consumido 3 dias/semana, marque $\mathbf{3}$

Se é consumido 3 vezes/mês, marque $\mathbf{T}$

Se é consumido quinzenalmente, marque $\mathbf{Q}$

Se raramente ou nunca consumido, marque $\mathbf{R}$

Visualização

(7) $6 \begin{array}{llllllll}5 & 5 & 4 & 3 & 2 & 1 & \mathrm{~T} & \mathrm{Q} \\ \mathrm{R}\end{array}$

$\begin{array}{lllllllll}7 & 6 & 5 & 4 & (3) & 2 & 1 & \mathrm{~T} & \mathrm{Q} \\ \mathrm{R}\end{array}$

$\begin{array}{lllllllllllll}7 & 6 & 5 & 4 & 3 & 2 & 1 & \text { (T) Q } & R\end{array}$

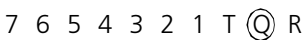

$\begin{array}{lllllllll}7 & 6 & 5 & 4 & 3 & 2 & 1 & \mathrm{~T} & \mathrm{Q}(\mathrm{R})\end{array}$

Se marcar $\mathbf{R}$, para algum alimento, identificar por qual das razões:

1 - Não gosta

2 - Não tem hábito

3 - Preço elevado

4 - Evita

Indicar também a quantidade ingerida, conforme o tamanho que mais se aproxime da porção, observado o álbum fotográfico: \begin{tabular}{|l|l|l|l|l|}
\hline A & B & C & D & E \\
\hline
\end{tabular}

Exemplo:

Freqüência consumo Porção do álbum Razão não consumo

Água:
(7)65 $4321 \mathrm{TQR}$
$A B$ (C) $D$
1234

Legenda:

Sbr - Colher de sobremesa rasa; Sbc - Colher de sobremesa cheia; SR - Colher de sopa rasa; SC - Colher de sopa cheia; Pq - Unidade, pequena; Gd - Unidade, grande. 
4 INQUÉRITO

4.1 CARNES E PESCADOS

Almôndega

A $B$ C $D$ D

Bife de boi

30456090150

A B C D E 11234

4285120165200

Carne cozida

A $B \quad C \quad D \quad E$

3050107180250

Carne moída

A B C D E

Frango assado

4182135180225

A B C D E

206590138230

Lingüiça

A B C D E

Peixe frito

446286110154

A B C D E

4580110156220

4.2 OVOS

Ovo frito

$1-2-3-4-5$ (unidades)

4.3 LEITE E DERIVADOS

Leite de Vaca

A B C D E

Leite em Pó

100165230330750

A B C D E

Leite em Pó (colheres)

$7 \quad 275481100$

Mussarela

$7654321 \mathrm{TQR}$

1 - 2 - 3 - 4 - 6 (fatias)

Queijo Minas

4.4 CEREAIS E FARINHAS

Angu

A B C D E

173045110164

A B C D E

Arroz

153070120200

A B C D E

4171110150233

Biscoito água e sal

A B C D E

Bolo básico

$828 \quad 65 \quad 100200$ 
Coxinha

$7654321 \mathrm{TQR}$

Coxinha (pq e gd)

$7654321 \mathrm{~T} \mathrm{QR}$

Farofa

$7654321 \mathrm{TQR}$

Lazanha

$7654321 \mathrm{TQR}$

Macarrão

$7654321 \mathrm{TQR}$

Pão de queijo

$7654321 \mathrm{TQR}$

Pão de queijo (pq gd)

$7654321 \mathrm{TQR}$

Pão francês

$7654321 \mathrm{TQR}$

Pão de forma

$7654321 \mathrm{TQR}$

Pastel de carne

$7654321 \mathrm{TQR}$

Pastel de carne (pq gd)

$$
7654321 \mathrm{TQR}
$$

Pipoca

$7654321 \mathrm{TQR}$

Pizza

$7654321 \mathrm{TQR}$

4.5 LEGUMINOSAS

Feijão cozido

$7654321 \mathrm{TQR}$

4.6 HORTALIÇAS GRUPO A

Alface

$7654321 \mathrm{TQR}$

Berinjela

$7654321 \mathrm{TQR}$

Couve crua

$7654321 \mathrm{TQR}$

Couve refogada

$7654321 \mathrm{TQR}$
A B C D E

$\begin{array}{llll}1 & 2 & 3 & 4\end{array}$

$20 \quad 42 \quad 75115155$

A B

22100

A B C D E

10224075100

A B C D E

120180240340440

A B C D E

50100130200280

A B C D

4085130250480

A B

$\begin{array}{llll}1 & 2 & 3 & 4\end{array}$

2550

$1 / 2-1-2-3-4$ (unidades)

$\begin{array}{lllll}25 & 50 & 100 & 150 & 200\end{array}$

$1 / 2-1-2-3-4$ (fatias)

$\begin{array}{lllll}25 & 50 & 75 & 100 & 125\end{array}$

A B C D E

205070100125

A B

2492

A B C D E

$153050 \quad 65 \quad 80$

$\begin{array}{llll}1 & 2 & 3 & 4\end{array}$

$\begin{array}{llll}1 & 2 & 3 & 4\end{array}$

$\begin{array}{llll}1 & 2 & 3 & 4\end{array}$

$\begin{array}{llll}1 & 2 & 3 & 4\end{array}$

1234

$85140190230 \quad 330$

$\begin{array}{lllll}A & B & C & D & E\end{array}$

$\begin{array}{llll}1 & 2 & 3 & 4\end{array}$

3971142200270

A $B \quad C \quad D \quad E$

$10 \quad 35 \quad 60 \quad 80100$

A B C D E

$3045 \quad 6075 \quad 105$

A B C D E

1020366080

A B C D E

1020366080 $\begin{array}{llll}1 & 2 & 3 & 4\end{array}$

$\begin{array}{llll}1 & 2 & 3 & 4\end{array}$

$\begin{array}{llll}1 & 2 & 3 & 4\end{array}$

$\begin{array}{llll}1 & 2 & 3 & 4\end{array}$ 
550 | R.L. SALES et al.

Couve-flor

$7654321 \mathrm{TQR}$

Pepino

$7654321 \mathrm{TQR}$

Tomate

$7654321 \mathrm{TQR}$

4.7 HORTALIÇAS GRUPO B

Cenoura

$7654321 \mathrm{TQR}$

Beterraba

$7654321 \mathrm{TQR}$

Vagem

$7654321 \mathrm{TQR}$

4.8 HORTALIÇAS GRUPO C

Batata baroa

$7654321 \mathrm{TQR}$

Batata frita:

$7654321 \mathrm{TQR}$

Mandioca cozida

$7654321 \mathrm{TQR}$

Mandioca frita

$7654321 \mathrm{TQR}$

4.9 FRUTAS GRUPO A

Melão

$7654321 \mathrm{TQR}$

Laranja

$7654321 \mathrm{TQR}$

4.10 FRUTAS GRUPO B

Abacaxi

$7654321 \mathrm{TQR}$

Banana

$7654321 \mathrm{TQR}$

Maçã

$7654321 \mathrm{TQR}$

Mamão

$7654321 \mathrm{TQR}$

Uva

$7654321 \mathrm{TQR}$
A B C D E

$\begin{array}{llll}1 & 2 & 3 & 4\end{array}$

3060103214321

A B C D E

22427485100

$\begin{array}{llll}1 & 2 & 3 & 4\end{array}$

A $B$ C D $\quad E$

15305075140

$\begin{array}{llll}1 & 2 & 3 & 4\end{array}$

A B C D E

$\begin{array}{llll}1 & 2 & 3 & 4\end{array}$

$\begin{array}{lllll}10 & 25 \quad 40 \quad 80130\end{array}$

A B C D E

14305285110

A B C D E

3775110140220

$\begin{array}{llll}1 & 2 & 3 & 4\end{array}$

1234

A B C D E

1234

6085120180240

A B C D E

$\begin{array}{llll}1 & 2 & 3 & 4\end{array}$

255080100150

A B C D E

306090130210

$\begin{array}{llll}1 & 2 & 3 & 4\end{array}$

A B C D E

306090130210

$\begin{array}{llll}1 & 2 & 3 & 4\end{array}$

A B C D E

$\begin{array}{llll}1 & 2 & 3 & 4\end{array}$

70140210280410

A B C D E

$\begin{array}{llll}1 & 2 & 3 & 4\end{array}$

90120189370500

A B C D E

75150300375540

$\begin{array}{llll}1 & 2 & 3 & 4\end{array}$

A B C D E

$\begin{array}{llll}1 & 2 & 3 & 4\end{array}$

75105120210225

A B C D E

$\begin{array}{llll}1 & 2 & 3 & 4\end{array}$

4080130160240

A B C D E

$\begin{array}{llll}1 & 2 & 3 & 4\end{array}$

70140280420580

A B C D E

$40 \quad 80120176350$
1234 
4.11 DOCES

Bombom

$7654321 \mathrm{TQR}$

Cajuzinho

$7654321 \mathrm{~T} Q \mathrm{R}$

Cajuzinho (pq gd)

$7654321 \mathrm{~T}$ Q R

Doce de Leite pastoso

$7654321 \mathrm{TQR}$

Doce de Leite pedaço

$7654321 \mathrm{TQR}$

Gelatina

$7654321 \mathrm{TQR}$

Goiabada

$7654321 \mathrm{TQR}$

Nescau

$7654321 \mathrm{TQR}$

Nescau (colheres)

$7654321 \mathrm{~T} \mathrm{QR}$

4.12 BEBIDAS E INFUSÕES

Café

$7654321 \mathrm{TQR}$

4.13 ÓLEOS E GORDURAS

Margarina

$7654321 \mathrm{TQR}$

4.14 DIVERSOS

Purê de batata

$7654321 \mathrm{~T}$ Q R

Salada de legumes com maionese

$$
7654321 \mathrm{~T} Q \mathrm{R}
$$

A B C D E

1234

$122030 \quad 60100$

A B C D E

1234

10204060120

A B

1234

822

A $\quad B \quad C \quad D \quad E$

356090120200

A B C D E

356090120200

A B C D E

$\begin{array}{llll}1 & 2 & 3 & 4\end{array}$

65130200330500

A B C D E

4560100120175

A B C D E

$816 \quad 324864$

Sbr Sbc SR SC

$\begin{array}{llll}4 & 10 & 7 & 21\end{array}$

1234

$\begin{array}{llll}1 & 2 & 3 & 4\end{array}$

1234

A B C D E

50100165240330

1234

A B C D E

$816 \quad 244080$

1234

A B C D E

254580135200

A B C D E

3570140210280
1234

$\begin{array}{llll}1 & 2 & 3 & 4\end{array}$ 
5 CONSUMO DE PADRÃO DIÁRIO

REFEIÇÃO

\begin{tabular}{|c|c|c|c|}
\hline \multirow{2}{*}{ Refeição } & \multirow{2}{*}{ Alimento } & \multicolumn{2}{|c|}{ Quantidade } \\
\hline & & Mc & Gramas \\
\hline & & & \\
\hline & & & \\
\hline & & & \\
\hline & & & \\
\hline & & & \\
\hline & & & \\
\hline & & & \\
\hline & & & \\
\hline & & & \\
\hline & & & \\
\hline & & & \\
\hline & & & \\
\hline & & & \\
\hline & & & \\
\hline & & & \\
\hline & & & \\
\hline & & & \\
\hline & & & \\
\hline & & & \\
\hline & & & \\
\hline & & & \\
\hline
\end{tabular}

6 OUTROS

(Alimentos normalmente consumidos pela pessoa, não incluídos no questionário)

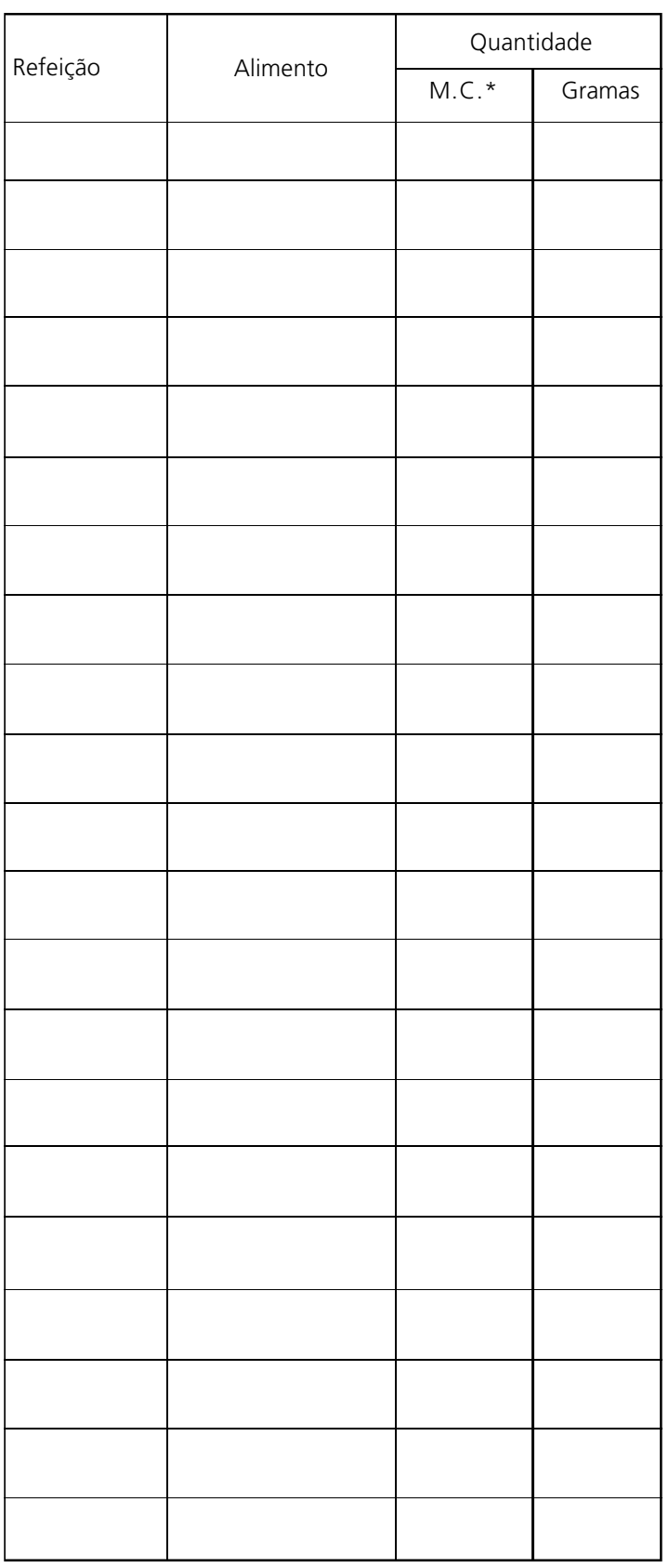

*MC: Medidas Caseiras 